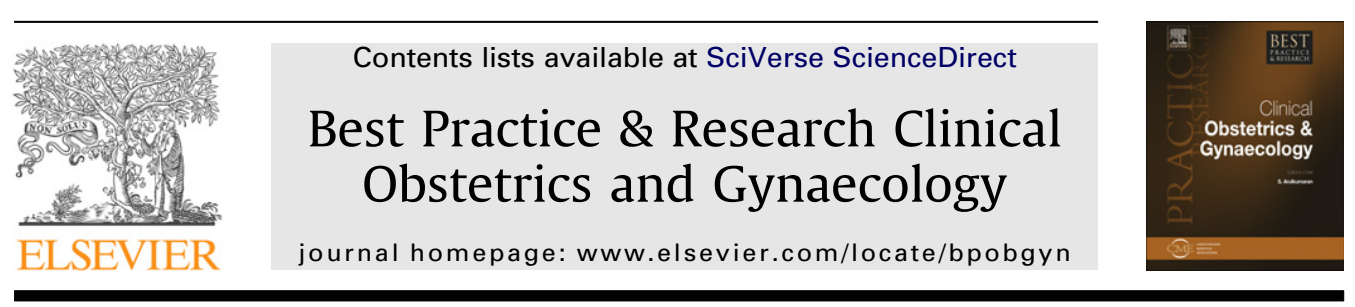

10

\title{
Fertility-preserving surgical procedures, techniques
}

\author{
Alejandra Martinez, MD ${ }^{\mathrm{a}, *}$, Mathieu Poilblanc, MD ${ }^{\mathrm{a}}$, Gwenael Ferron, MD ${ }^{\mathrm{a}}$, \\ Mariolene De Cuypere, $\mathrm{MD}^{\mathrm{a}}$, Eva Jouve, $\mathrm{MD}^{\mathrm{a}}$, Denis Querleu, $\mathrm{PhD}^{\mathrm{a}} \mathrm{b}$ \\ a Surgical Oncology Department, Claudius Regaud Comprehensive Cancer Center, 20-24, Rue du Pont-Saint-Pierre, \\ 31052 Toulouse, France \\ ${ }^{\mathrm{b}}$ Department of Gynecologic Oncology, McGill University Health Center, Montreal, Quebec, Canada
}

\section{Keywords:}

fertility preservation

surgical technique

gynaecological cancer

radical trachelectomy

ovarian transposition
As a result of the trend toward late childbearing, fertility preservation has become a major issue in young women with gynaecological cancer. Fertility-sparing treatments have been successfully attempted in selected cases of cervical, endometrial and ovarian cancer, and gynaecologists should be familiar with fertilitypreserving options in women with gynaecological malignancies. Options to preserve fertility include shielding to reduce radiation damage, fertility preservation when undergoing cytotoxic treatments, cryopreservation, assisted reproduction techniques, and fertility-sparing surgical procedures. Radical vaginal trachelectomy with laparoscopic lymphadenectomy is an oncologically safe, fertility-preserving procedure. It has been accepted worldwide as a surgical treatment of small early stage cervical cancers. Selected cases of early stage ovarian cancer can be treated by unilateral salpingo-ophorectomy and surgical staging. Hysteroscopic resection and progesterone treatment are used in young women who have endometrial cancer to maintain fertility and avoid surgical menopause. Appropriate patient selection, and careful oncologic, psychologic, reproductive and obstetric counselling, is mandatory. (c) 2012 Elsevier Ltd. All rights reserved.

\section{Cervical cancer}

Fertility preservation is an important component of the overall quality of life of cervical cancer survivors. Excisional cone biopsies alone can be considered in women with stage IA1 cervical cancer

\footnotetext{
* Corresponding author. Tel: +33 5614242 42; Fax: +33561424117.

E-mail address: martinez.alejandra@claudiusregaud.fr (A. Martinez).
} 
and no lymphovascular space invasion or involvement. Standard treatment of women with more extensive disease confined to the uterus is radical hysterectomy with pelvic lymphadenectomy, which eliminates any possibility for future pregnancy. Selected women can be candidates for a radical trachelectomy procedure (conisation) with conservation of the uterus and ovaries. The procedure can be carried out with a cold knife, laser, or electrosurgical loop.

\section{Radical trachelectomy}

Radical trachelectomy has emerged as a valuable fertility-preserving treatment option for young women with early stage cervical cancer. Accumulating data confirm that, overall, oncological outcome is safe and obstetrical results promising. ${ }^{1}$ Radical vaginal trachelectomy (RVT) with laparoscopic lymphadenectomy is a fertility-preserving procedure that has recently gained worldwide acceptance as a method of surgically treating small invasive cervical cancers. Since the original description of RVT by Daniel Dargent et al. ${ }^{44}$ in 1994, over 1000 cases of women having undergone this technique have been reported, ${ }^{1-4}$ with over 250 live births reported in women after having this procedure. Patient selection for the procedure is extremely important for success. Accepted criteria for radical trachelectomy are women with an important desire for future fertility, squamous cell carcinoma, adenocarcinoma or adenosquamous, with exclusion of unfavourable histology, stage IA1 with lymphovascular invasion, Ia2, or IB1 of less than $2 \mathrm{~cm}$, tumour limited to the cervix, and no evidence of lymphatic spread.

Morbidity associated with RVT is low, with tumour recurrence rates of between 4.2 and $5.3 \%$, and mortality rates between 2.5 and 3.2\%. Risk factors for recurrence are lesion size of more than $2 \mathrm{~cm}$, lymphovascular involvement, and unfavourable histology (i.e. small-cell neuroendocrine tumours). ${ }^{5}$ Pregnancy rates vary from $41-79 \%$, risk of second trimester miscarriage is twice the rate of general population, and preterm delivery is about $30 \%$, but only $12 \%$ with significant prematurity. Infertility is
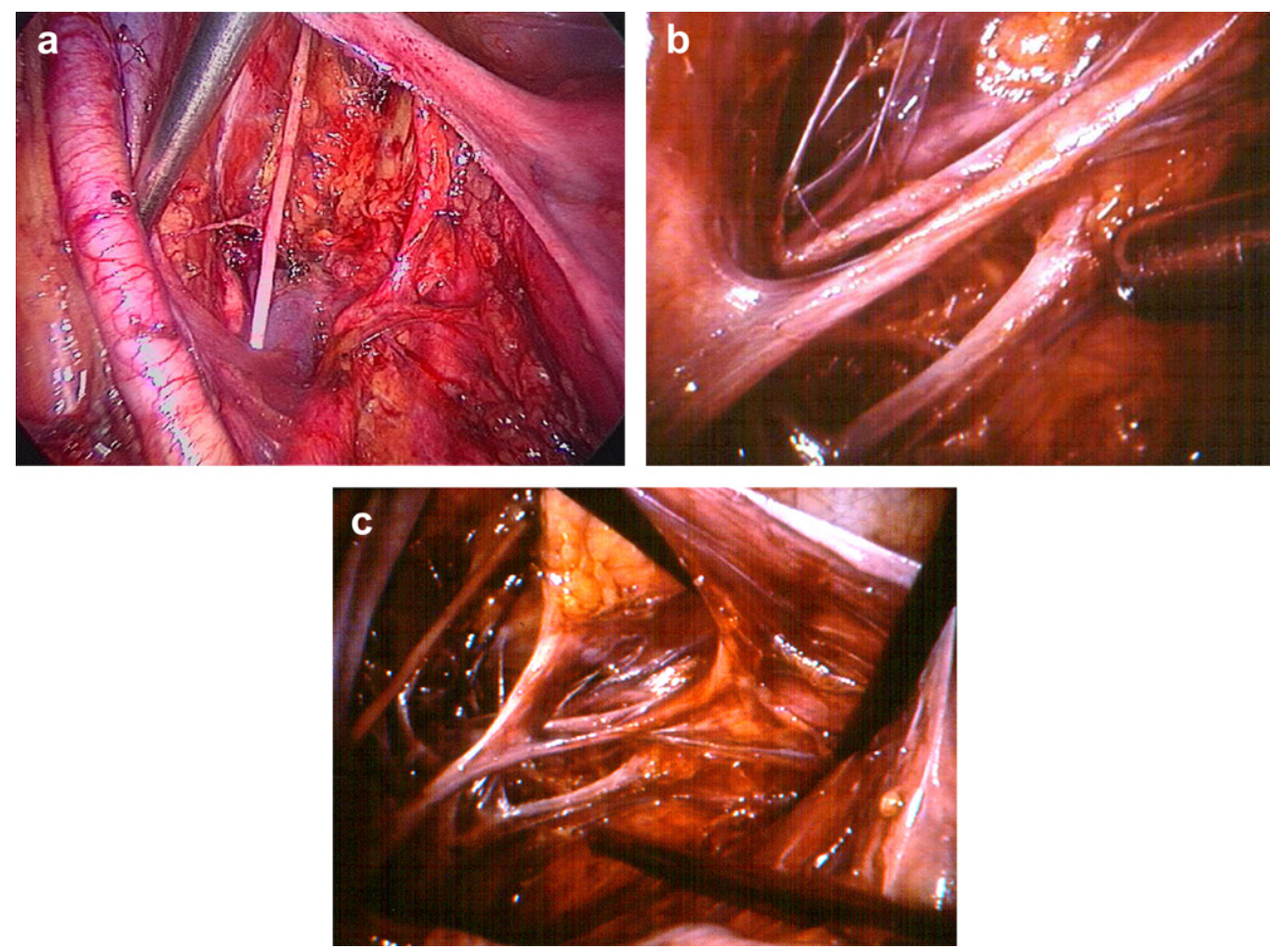

Fig. 1. (a) Pelvic lymphadenectomy; (b) and (c) paracervical lymphadenectomy. 


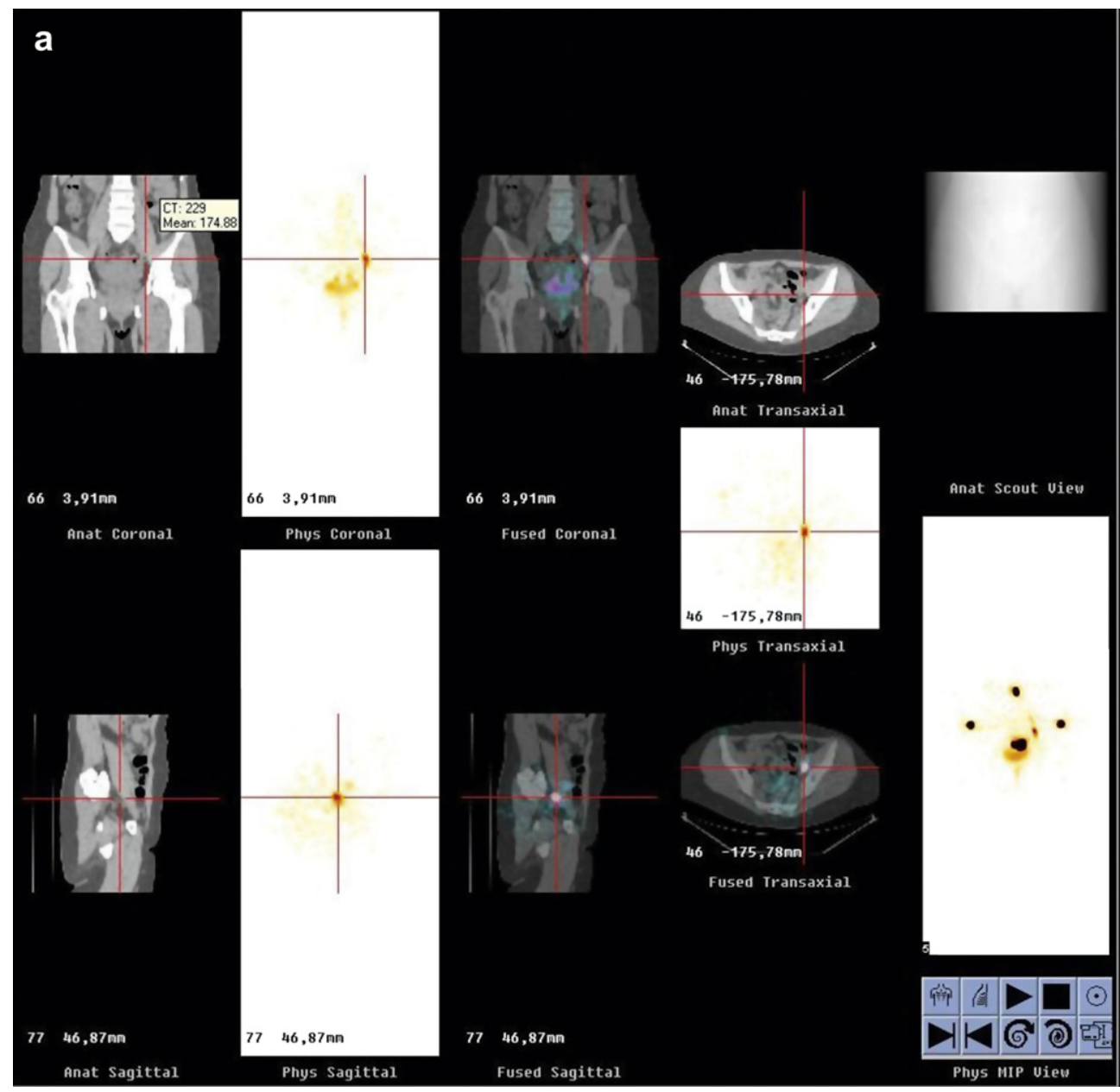

Fig. 2. (a) Single photon emission-computed tomography scan of sentinel lymph node localised at the left external iliac area (b-d); blue and hot external iliac sentinel lymph nodes.

found in up to one-third of women, and is related in most cases to cervical factors (i.e. cervical stenosis, decreased cervical mucus, surgical adhesion formation and subclinical salpingitis). ${ }^{1,2,6,7}$

Radical trachelectomy can be carried out vaginally, ${ }^{1,3,7,8}$ abdominally, ${ }^{9}$ laparoscopically, ${ }^{10,11}$ and recently a robotic approach has been used. ${ }^{12-14}$ Radical trachelectomy, via a trans-sacral approach,has also been described in a woman with a history of rectal resection and radiotherapy for rectal cancer, who had unacceptable risks associated with a laparotomic approach. ${ }^{15}$

\section{Exploration}

The abdomen and pelvis are examined systematically at the beginning of the operation by inspecting the peritoneal cavity, and includes a detailed examination of the fallopian tubes and ovaries. Frozen section of any suspicious lesion is required before starting the procedure, which should be abandoned in case of metastatic disease.

\section{Pelvic lymphadenectomy}

A laparoscopic, or open pelvic lymphadenectomy for abdominal radical trachelectomy, is carried out before the trachelectomy procedure (Fig. 1). Pelvic nodes from the common iliac bifurcation 

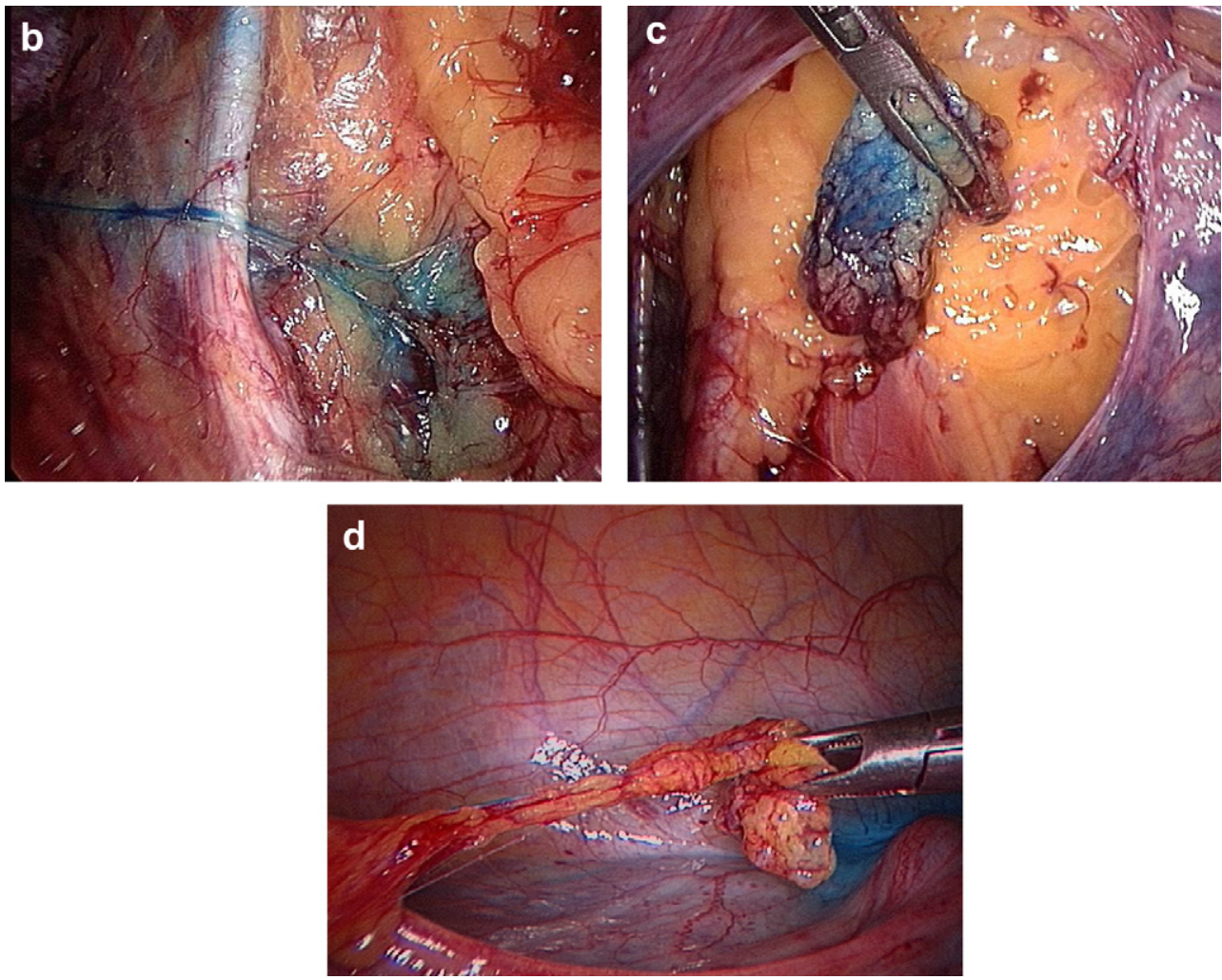

Fig. 2. (Continued).

proximally to the circumflex vein distally, including the pelvic nodes from the external iliac, internal iliac, and obturator regions, are removed and sent for intraoperative histology. In Fig. 1a, a pelvic lymphadenectomy has been carried out. Iliac vessels and obturator nerve are completely exposed. In Fig. 1b and c, a paracervical lymphadenectomy has been carried out. Sentinel lymph-node biopsy with frozen section is possibly the best and most efficient option to evaluate node status before starting the trachelectomy procedure (Fig. 2). A sentinel lymph-node biopsy also increases the detection rate of lymph-node metastases by identifying unusual locations of sentinel lymph nodes that are not removed by standard lymphadenectomy. The procedure is abandoned if positive nodes are found and paraaortic lymph-node sampling is carried out. In Fig. 2a, a single photon emission-computed tomography shows sentinel lymph nodes localised at the left external iliac area; Fig. 2b, $c$ and d show blue and hot external iliac sentinel lymph nodes.

\section{Vaginal trachelectomy}

Vaginal trachelectomy is begun by delineating an adequate vaginal margin of around $1-2 \mathrm{~cm}$. Six to eight Kocher are placed circumferentially, and dilute xylo adrenaline solution is injected under the vaginal mucosa to reduce bleeding and facilitate dissection. The vaginal mucosa is incised, and the anterior and posterior aspects of the vaginal incision are folded together. Kocher clamps are removed and Krobach clamps are placed horizontally over the vaginal mucosa (Fig. 2). In Fig. 3, vaginal mucosa has been incised after xylo-adrenaline injection. 


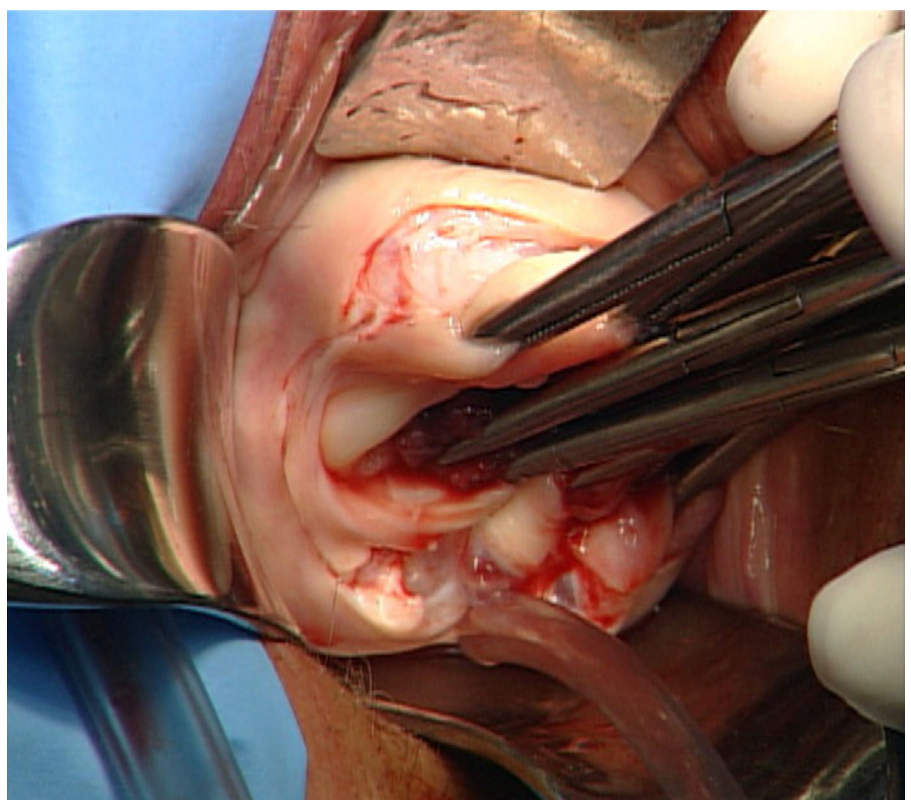

Fig. 3. Vaginal mucosa has been incised after xylo-adrenaline injection.

\section{Development of retroperitoneal spaces}

Rectovaginal space. The posterior cul-de-sac is opened posteriorly, the rectovaginal space is created and the proximal part of the rectovaginal ligament is divided.

Prevesical space. The specimen is tracted downwards and the prevesical space is entered and developed by sharp dissection.

Paravesical space. Two Kocher claps are placed at 1 and 3 o'clock positions on the vaginal mucosa, and Metzenbaum scissors are introduced in an antero-lateral direction to enter and develop the paravesical spaces on both sides. Once the prevesical and paravesical spaces are developed, the bladder pillar is dissected and isolated from the cardinal ligament. The ureter is identified by palpation at the midportion of the bladder pillar, pushed cephalad enabling safe transection of the uterovesical ligament distal to the ureter (Fig. 4). Here, the paravesical space has been opened, the knee ureter has been identified, and the bladder pillar can be transacted, avoiding ureteral injury.

\section{Parametrial dissection}

Midportion of the parametrium is clamped or coagulated and divided. Only the descending branch of the uterine artery, the cervicovaginal branch, is coagulated or ligated and divided without disturbing the remaining blood supply to the uterus.

\section{Amputation of the cervix}

The cervix is transected about $1 \mathrm{~cm}$ below the internal cervical orifice, and the specimen is removed after vaginal section (Fig. 5). Fig. 5a and b show the cervix transected below the internal oriface. The endocervical canal can be identified.

A prophylactic permananent cerclage is placed at the level of the internal oriface to avoid cervical incompetence. A No. 8 French rubber catheter is inserted into the remaining cervix to avoid stenosis. In 


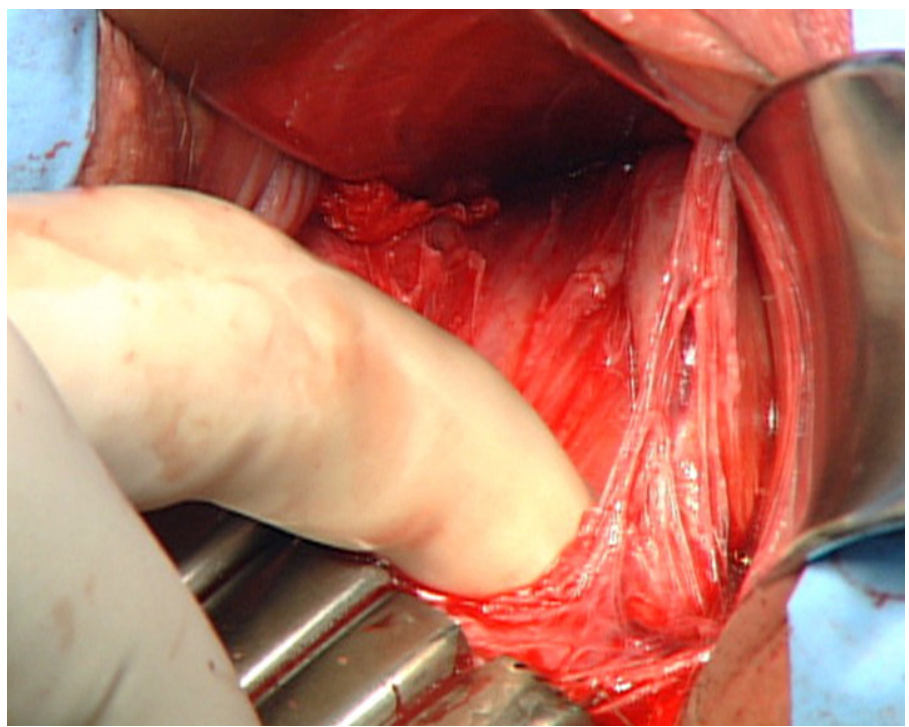

Fig. 4. Opened paravesical space and identification of knee ureter; the bladder pillar can be transacted, avoiding ureteral injury.
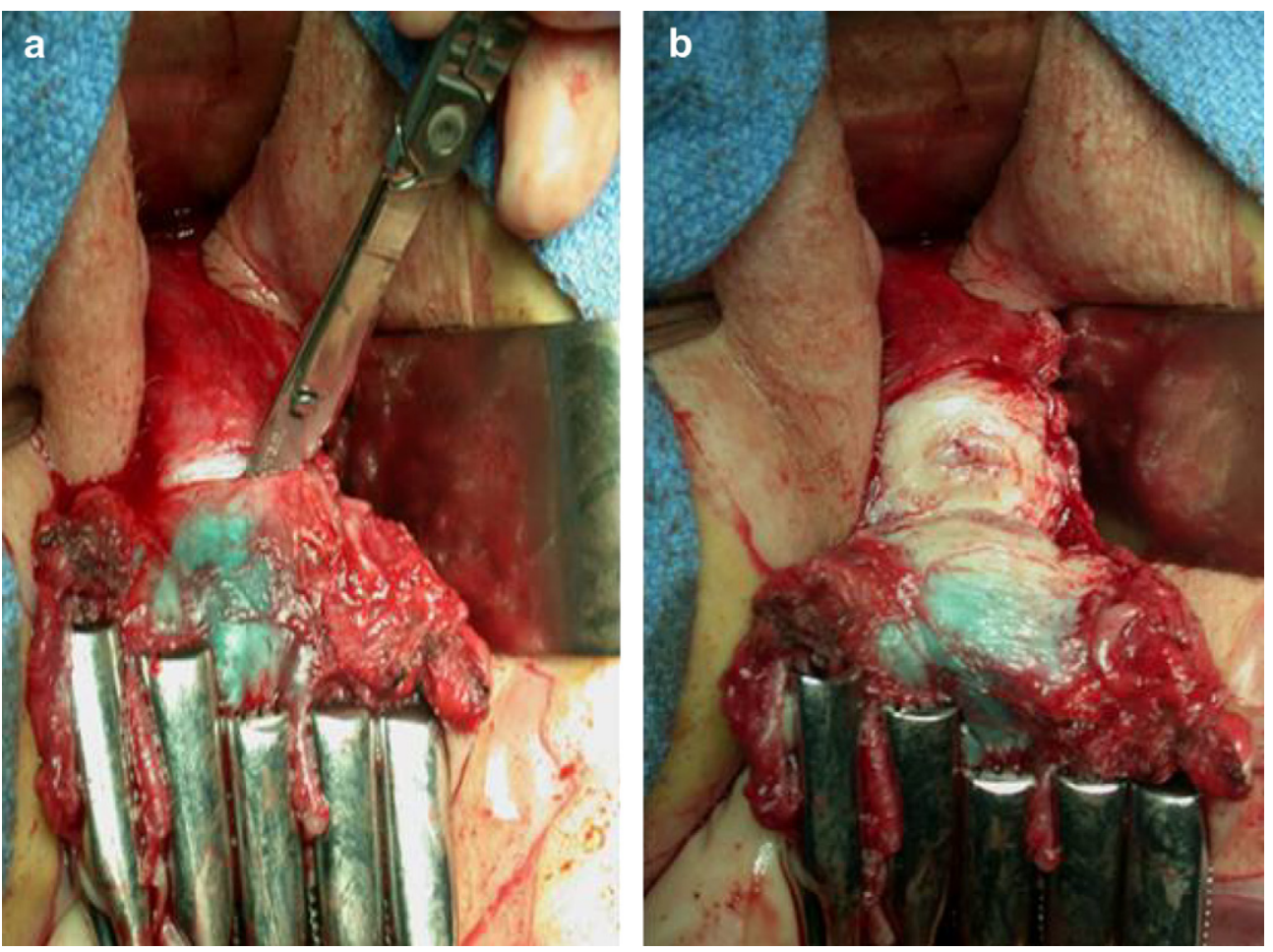

Fig. 5. ( $\mathrm{a}$ and $\mathrm{b}$ ) The cervix has been transected below the internal orifice. The endocervical canal can be identified. 
Fig. 6, a No. 8 French rubber has been introduced into the endocervical canal to ensure permeability. The cervical stump is readapted and sutured to the vaginal mucosa.

A frozen section of the superior margin of the cervix is carried out to ensure safe negative endocervical margins. When the upper limit of the tumour is less than $5 \mathrm{~mm}$, removing another $3-5 \mathrm{~mm}$ of the residual cervix is recommended to improve margin of tumour clearance. When the upper margin is involved, a radical hysterectomy should be carried out. Women should always be informed of this possibility before surgery.

A laparoscopic approach is carried out at the end to verify haemostasis and the integrity of pelvic structures.

\section{Abdominal trachelectomy}

Abdominal radical trachelectomy is identical to radical hysterectomy, with preservation of the uterine fundus by separation of the uterine cervix and the parametrium at the level of the internal os. ${ }^{4,9}$

\section{Development of retroperitoneal spaces}

The procedure begins by opening the retroperitoneal space through the round ligament laterally. The broad ligament of the uterus is incised anteriorly down to the bladder reflection. The posterior leaf is incised above the psoas muscle in a cephalad direction, lateral and parallel to the ovarian vessels. The pararectal and paravesical spaces are developed using blunt dissection to expose the ureter and the iliac vessels on each side of the pelvis.

Paravesical space. Non-traumatic forceps are used. The surgeon develops the paravesical space, bound by the umbilical obliterated artery medially, the external iliac vein laterally, the ventral aspect of the cardinal ligament posteriorly, the pubic symphisis anteriorly, and the obturator fossa and muscle inferiorly. The posterior peritoneal layer with the ureter attached is retracted towards the midline, and the pararectal space is developed by separating the connective tissues between the internal iliac artery and the posterior leaf of the broad ligament.

Pararectal space. The pararectal space is bounded by the rectum and ureter medially, the sacrum dorsally, the pelvic sidewall and iliac vessels laterally, and the cardinal ligament anteriorly.

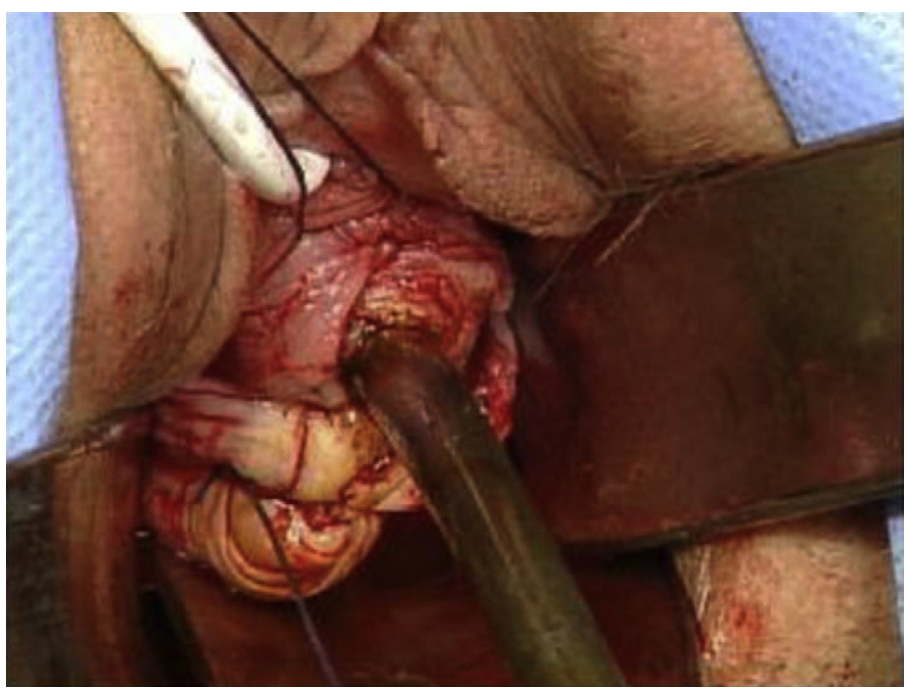

Fig. 6. A No. 8 French rubber has been introduced into the endocervical canal to ensure permeability. 


\section{Bladder mobilisation}

The peritoneal incision is continued down to the side of the bladder, the vesicouterine peritoneal fold is incised, and the bladder is completely detached from the mid-sagital cervix and the proximal third of the vagina. This dissection can be accomplished with either scissors or electrocautery. Cervical anterior margin is verified to ensure no disease spreads beneath the bladder.

\section{Dissection of the ureter and division of the uterine artery}

The ureter is dissected from its entry into the broad ligament to its intramural portion in the bladder. The ureter is first isolated from the posterior leaf of the broad ligament and displaced laterally by inserting the tip of the scissors between the ureter and the peritoneum proximal to the uterosacral ligament. An arterial branch, 2-3 $\mathrm{mm}$ below the common iliac bifurcation, should be identified and divided.

Between the paravesical and pararectal space, the thick bundle of parametrial tissue is exposed. The uterine artery is identified, ligated, coagulated, or clipped and divided as it crosses the ureter. The uterine vein running below the artery is then identified, divided and ligated. The artery is tracked upwards and dissected free from the underlying paracervix and from the ureter to expose the ureteral tunnel of Wertheim. The ureter is separated from the medial leaf of the broad ligament until it enters the parametrial tunnel. A ureteral branch from the uterine artery encountered at this point of the dissection may be coagulated with a bipolar device or clipped.

A right-angle clamp is introduced into the proximal tunnel on the superomedial surface of the ureter. The anterior leaf of the vesicouterine ligament is divided to progressively deroof the ureter to the bladder. The ureter is then rolled laterally and separated from its loose attachments to the dorsal aspect of the vesicouterine ligament, permitting to transect it. Troublesome venous bleeding can occur at this stage of the operation, and small vessels should be ligated or clipped to avoid bleeding.

Parametrial dissection. After unroofing the ureter, the parametrium is completely exposed and retracted while the ureter is displaced medially to allow division of the cardinal ligament. The paracervix is then clamped, coagulated with a bipolar device, clipped, or divided and suture ligated at the level of the

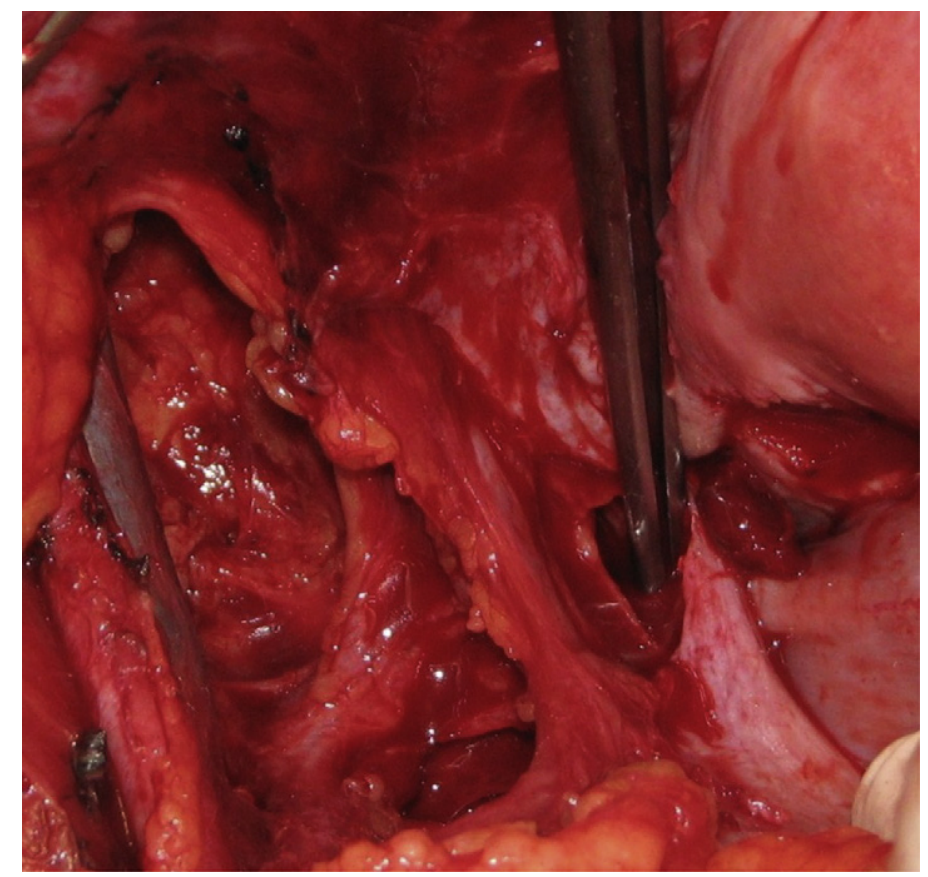

Fig. 7. Pararectal and paravesical spaces have been created and the parametrium is completely exposed. 
ureteral tunnel. The deep component of the paracervix, caudal to the deep uterine vein, is not divided to preserve neural structures. In Fig. 7, pararectal and paravesical spaces have been created and the parametrium is completely exposed.

Dissection of the uterosacral ligaments. The posterior leaf of the broad ligament, from which the ureter has been removed, is transected from its superior margin to the uterosacral ligament. The uterus is anteflexed, the rectum retracted towards the sacral promontory, and the cul-de-sac is incised to open and develop the rectovaginal space with blunt dissection. The uterosacral ligaments are clamped, ligated, or coagulated and divided halfway along their length.

Vaginal resection. Paracolpium is completely exposed to permit an optimal tailoring of the vaginal cuff. The vagina is transected $10 \mathrm{~mm}$ distal to the limit of the anterior cervical lip. The uterine fundus with preserved adnexal attachments along with the trachelectomy specimen and is placed on the pelvis.

Uterine section. The lower uterine margin is estimated and claps are placed at the level of the internal cervical orifice. The upper endocervix is separated from the isthmic part of the uterus at about $5 \mathrm{~mm}$ distal to the internal orifice. ${ }^{9}$ After specimen removal, a shave disc margin of the remaining cervix is sent for frozen section analysis. If the upper endocervical margins are positive, a total radical hysterectomy must be carried out. In most cases, planned operation continues by closing the peritoneum of the cul-de-sac, and the vaginal mucosa is sutured to the remaining cervical stump. A prophylactic cerclage with a non-reabsorbable suture is carried out.

Laparoscopic and robotic radical trachelectomy

The woman is placed in a dorsal lithotomy position, with arms tucked at the sides.

\section{Trocar placement for laparoscopic approach}

A camera is inserted through the umbilicus, and three operative trocars are placed under direct vision; two 5-mm ancillary trocars are inserted in the lower quadrants lateral to the epigastric vessels, and a $10 \mathrm{~mm}$ trocar is placed suprapubically in the midline.

\section{Trocar and instrument placement for robotic approach}

The woman is placed in a dorsal lithotomy position. A 12-mm trocar is placed on the left upper quadrant, $2 \mathrm{~cm}$ below the costal margin at the mid-clavicular line, used for insuflation and for the surgical assistant during the procedure. A 12-mm umbilical trocar for the robotic camera, and the two robotic trocars placed $8-10 \mathrm{~cm}$ lateral to the umbilical trocars, are inserted under direct vision. The third robotic trocar is placed $8 \mathrm{~cm}$ lateral to the right robotic trocar. The robotic instruments are then placed: an Endowrist PK grasper (Intuitive Inc., Sunnyvale, CA) on the left robotic arm, an Endowrist monopolar spatula or scissors (Intuitive Inc., Sunnyvale, CA) on the right robotic arm, and an Endowrist bipolar grasper (Intuitive Inc., Sunnyvale, CA) on the third robotic arm. The robotic system is then docked.

\section{Prevesical and paravesical space}

The assistant tracts the round ligament close to the uterine horn to the midline with atraumatic forceps. Round ligament is divided using bipolar coagulation and scissors or other haemostatic devices, such as LigasSure ${ }^{\mathrm{TM}}$ or Ultracision ${ }^{\circledR}$, allowing access to the retroperitoneum between the two leaves of the broad ligament. Anterior and posterior leafs of the broad ligament are divided with monopolar scissors. The anterior peritoneal incision is continued to the prevesical peritoneum at the level of the cervicouterine junction.

The assistant tracts the uterus towards the sacral promontorium. Prevesical peritoneum and bladder are tracted upwards, and the space between the anterior wall of the vagina and the posterior wall of the bladder is developed. The bladder is dissected sharply from the vagina and pushed caudally with the help of monopolar scissors. Placement of a uterine manipulator helps mobilisation of the upper part of the vagina from the bladder. 


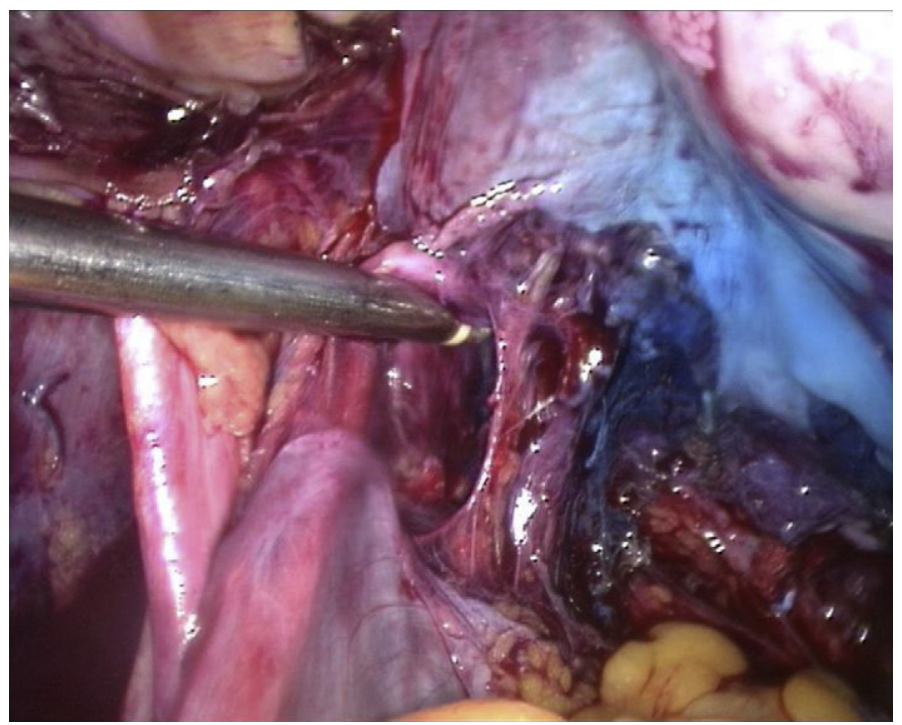

Fig. 8. Rectovaginal ligaments are exposed for their section.

\section{Pararectal space}

The posterior lateral peritoneal incision is continued to the level of the uterosacral ligaments. The ureters are dissected free from the medial peritoneal layer at the level of the sacrouterine ligaments down to their entry into the parametrial tissue. The posterior leaf of the broad ligament is tracted with grasp forceps and the space between the rectovaginal ligaments, and the mesoureter is opened with blunt dissection. The rectum is pulled cranially to open horizontally the peritoneum of the Douglas pouch, the rectovaginal space developed by blunt dissection, and the uterosacral ligaments divided. In Fig. 8, rectovaginal ligaments are exposed for their section.

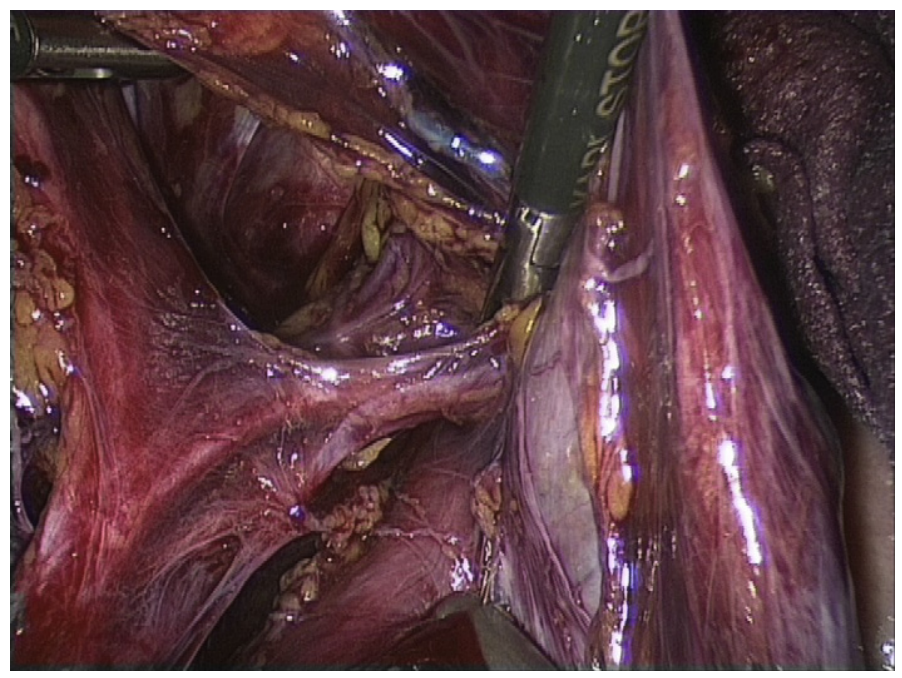

Fig. 9. Paravesical and pararectal spaces have been opened and paracervical tissue is exposed. 


\section{Parametrial division}

The uterine vessels are coagulated and divided or sealed with an endoscopic vessel-sealant device. In Fig. 9, paravesical and pararectal spaces have been opened and paracervical tissue is exposed. The medial edge of the uterine artery is grasped and tracted medially to expose the ureter roof. Dissection of the ureter from the vesicouterine ligament (ureteral tunnel) can be accomplished by using an endoscopic right angle dissector. Ureters are dissected bilaterally to their insertion into the bladder with lateral mobilisation. With the ureter suspended ventrally and laterally, the remaining paravaginal tissues are divided. Colpotomy is carried out circumferentially using monopolar scissors or hook. A glove is placed into the vagina to prevent leakage. Finally, the cervix is transected $1 \mathrm{~cm}$ below the isthmus, and the specimen removed vaginally. Prophylactic cerclage is carried out before placing vaginal interrupted sutures to the uterus, EndoWrist ${ }^{\circledR}$ Mega Needle is used for the robotic approach.

\section{Postoperative care and complications}

A Foley catheter is placed in all women for $48 \mathrm{~h}$ after the procedure. Postoperative bladder function is assessed at day 2 by measuring the post-void residual urine volume. If it is higher than $50 \mathrm{ml}$, the residual urine volume is measured after each miction, and is stopped when obtaining two post-void residual urine volumes of less than $100 \mathrm{ml}$ or one post-void residual urine volume less than $50 \mathrm{ml}$. In cases of urinary retention, patients are discharged with home self-intermittent catheterisation.

Peroperative morbidity of trachelectomy is similar to radical hysterectomy. Intraoperative complications include injury to the bladder, rectum, and neurological damage to the obturator or the autonomic nerves. Most frequent postoperative complication is urinary retention, other associated complications are urinary leakage, cervical stenosis, or ileus.

\section{Neoadjuvant chemotherapy}

Tumour size larger than $2 \mathrm{~cm}$ is associated with negative prognostic factors, such as deep stromal invasion, lymphovascular space invasion, tumour embols to the parametria or micrometastases, and is associated with a higher risk of recurrence, even in women undergoing radical surgery. Neoadjuvant chemotherapy is an emerging option for fertility-preserving surgery in women with stage IB1-2 or IIA1 tumours, with tumour volume of $2 \mathrm{~cm}$ or more to render women eligible for a conservative surgical approach. Fig. 10 shows a magnetic resonance imaging scan of a $3-\mathrm{cm}$ cervical tumour.

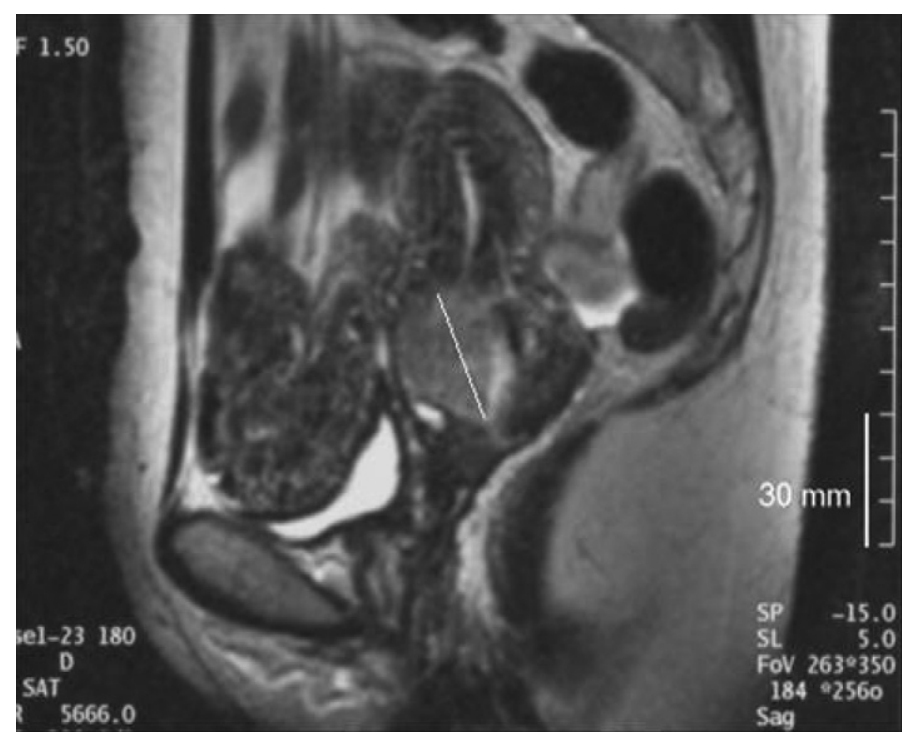

Fig. 10. A magnetic resonance imaging scan of a $3-\mathrm{cm}$ cervical tumour. 
Neoadjuvant chemotherapy improves surgical margins, permits a more conservative endocervical tissue resection and potentially offers better obstetrical outcomes. ${ }^{16}$ Tumour response to neoadjuvant chemotherapy is a strong independent predictor of survival, and can be used to follow or abandon a conservative surgical approach in cases of poor chemotherapy response. This strategy was first described by Plante et al. ${ }^{20}$ in three women with lesions measuring between 3 and $4 \mathrm{~cm}$ who had excellent responses to neoadjuvant chemotherapy followed by radical trachelectomy.

\section{Cone biospy or simple trachelectomy}

Maneo et al. ${ }^{17}$ assessed preoperative chemotherapy with cisplatin, paclitaxel and ifosfamide for reducing tumour volume followed by conisation alone and pelvic lymphadenectomy in 21 women desiring to preserve fertility with stage IB1 tumours less than $3 \mathrm{~cm}$, with no uterine involvement and no lymph-node metastasis. ${ }^{17}$ This strategy is based on limited parametrial invasion, less than $1 \%$ in women with tumour diameter less than $2 \mathrm{~cm}$, stromal invasion less than $10 \mathrm{~mm}$, and negative lymph nodes. ${ }^{18}$ Complete pathologic response was obtained in five women. Cervical cerclage was planned at the end of the first trimester in all women, and was removed before delivery allowing vaginal delivery. Only four women had neoplastic residue infiltrating deeper than $3 \mathrm{~mm}$ after neoadjuvant treatment and no invasive relapses were reported. Other groups have used similar protocols of three cycles of neoadjuvant chemotherapy with cisplatin, paclitaxel and ifosfamide (or epirubucine for adenocarcinomas) followed by conisation alone or with pelvic lymphadenectomy for selected women with tumours less than $3 \mathrm{~cm}$ with safe oncological outcome. No recurrences took place in the 11 women in the study by Landoni et al. ${ }^{19}$

About 30 women treated with neoadjuvant chemotherapy followed by conservative surgery have been reported. Disease recurred in three women treated with conisation as surgical strategy. ${ }^{16}$

Alkylating agents can be detrimental to ovarian follicles and are associated with ovarian fibrosis, which can lead to ovarian failure. ${ }^{20}$ Less gonadotoxic regimens should be evaluated in the future.

Larger studies and long-term data concerning safety, efficacy, and reproductive outcome are necessary to validate and define the indications for this conservative approach.

\section{Ovarian transposition in cervical cancer}

Since the early 1970s, many investigators have shown that ovarian transposition by laparotomy in women undergoing pelvic irradiation may allow preservation of ovarian function. Nowadays, this procedure enables future ovarian stimulation with the production of numerous eggs, which can later be retrieved under ultrasound guidance from the relocation site.

With advances in minimally invasive surgery, ovarian transposition is actually carried out laparoscopically. ${ }^{21-23}$ This approach is now preferred, allowing a short hospital stay and faster recovery, and preventing any delay in starting radiation therapy.

Different surgical techniques have been proposed for ovarian transposition (e.g. fixation of the ovary in the pouch of Douglas, ${ }^{24}$ lateral subcutaneous fixation, intraperitoneal or retroperitoneal fixation to the muscles psoas, and fixation in the paracolic gutter). ${ }^{25-27}$ In cases of gynaecological cancer, the most adapted technique is, in our experience, ovarian fixation to the paracolic gutter. Morice et al., ${ }^{30}$ studied bilateral laparoscopic ovarian transposition in women undergoing radiation therapy for pelvic tumours. The transposition was carried out in paracolic gutters of 95 women. They described a rate of ovarian preservation, in terms of function, as high as $89 \%$ in women undergoing postoperative vaginal brachytherapy, and $60 \%$ for women treated with brachytherapy and pelvic radiation.

Steps of the classical procedure are well-described and they are similar in laparoscopic and laparotomic approach. The objective is to place ovaries out of the radiation field, generally at least $3 \mathrm{~cm}$ above the upper border. $^{28}$

Only normal ovaries can be transposed, and the first step of the procedure is a careful visual inspection of the aspect, structure and size of the ovaries. A traction of the round ligament creates a tension in the peritoneum and the infundibulopelvic ligament. The retroperitoneal space is opened by incising the peritoneum on the side of the infundibulopelvic ligament. The ureter is controlled and the posterior wall of the broad ligament is incised, just behind the infundibulopelvic ligament in the 
avascular area. The peritoneum must be dissected carefully in order to preserve vascularisation supply and prevent any kinking after transposition. In order to mobilise the ovaries completely, the dissection of the ovarian vessels must take place up to the level of the aortic bifurcation.

The utero-ovarian ligament is then coagulated and cut off at its uterine origin. Some surgeons separate the fallopian tubes from the ovary through the mesovarium to transpose only the ovary. At our institution, we transpose both ovary and fallopian tube after tubal section at the uterine corn (Fig. 11). Here, the right adnexa has been mobilised out from the pelvis and transposed to the right paracolic gutter after section of the utero-ovarian ligament. At this point, the ovarian vessels could be turned laterally with a sufficient angle to maintain appropriate blood supply, ovaries are moved away from the radiation field and transposed to paracolic gutters. The ovary is transposed and fixed lateral and above the psoas muscle with a non-absorbable suture. The upper and lower poles of the ovaries are marked with hemoclips, allowing their localisation during radiation therapy.

Some surgeons described other techniques of fixation with confection of a retroperitoneal tunnel. After separating the adnexa from the uterus, a retroperitoneal tunnel is developed from the pelvis to the transposition opening laterally. The adnexa are moved through this tunnel, avoiding torsion of the vessels, and are brought through the opening back into the peritoneum. ${ }^{29}$

Complications of ovarian transposition are rare and include chronic ovarian pain, and metastasis to transposed ovaries and port sites. ${ }^{28}$ In their study, Morice et al. ${ }^{30}$ described 95 women who had experienced several types of complications, including one woman with ovarian metastasis 3 years after completing treatment, 22 cases of ovarian cysts, three cases of chronic abdominal pain, and one case of mechanical bowel obstruction resulting from the formation of a band adhesion between the omentum and the transposed ovary. The investigators concluded that ovarian transposition is a safe and effective procedure for the preservation of ovarian function. ${ }^{30}$

\section{Endometrial cancer}

The management of young women with early endometrial cancer is challenging. In order to preserve fertility, a conservative approach can be offered to young women with grade 1 endometrial adenocarcinoma, with no evidence of lymph-node metastasis or myometrial invasion. ${ }^{28,31}$ This option, however, is an exception, and women must be informed that numerous questions remain unresolved. The main issue of fertility-preserving strategy in the field of endometrial cancer is the safety of this option and the risk of occult ovarian tumour. The risk of disease progression during conservative

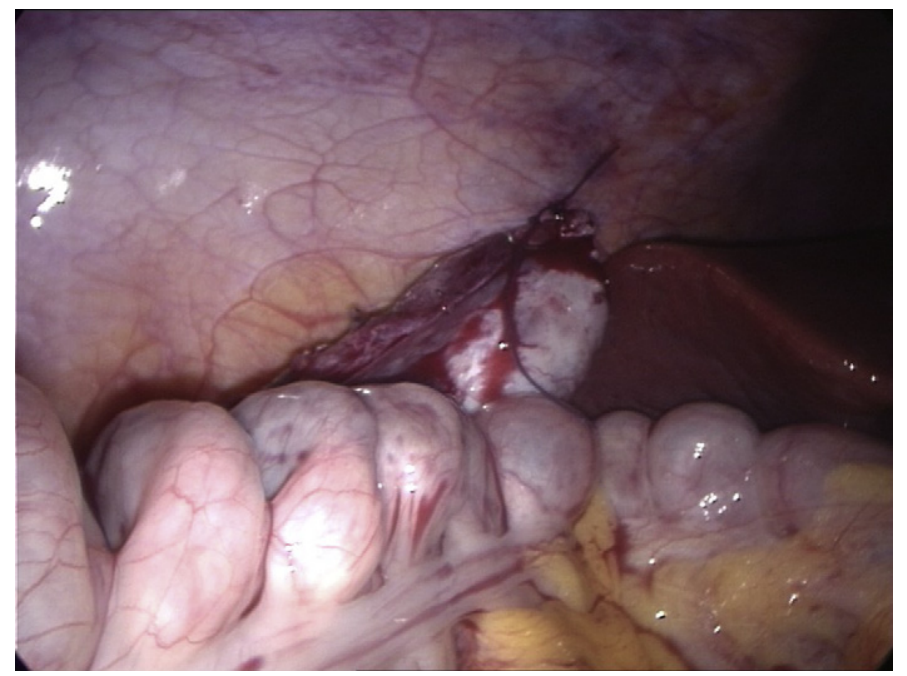

Fig. 11. Right adnexa has been mobilised out from the pelvis and transposed to the right paracolic gutter after section of the uteroovarian ligament. 
management of grade 1 endometrial carcinoma is as high as 5-6\% ${ }^{32-34}$ Endometrial cancer diagnosed at a younger age harbours the additional risk of cancers associated with Lynch syndrome as well as synchronous or metachronous ovarian cancers. In this context, the place of ovarian examination by laparoscopy is primordial in pre-therapeutic evaluation. In the case of grade 1 , stage $1 \mathrm{~A}$ endometrial carcinoma with macroscopic normal ovaries, the risk of occult ovarian tumour is about $1 \%{ }^{35,36}$

The prognosis for young women with endometrial cancer is good (i.e. well-differentiated lesion with minimal or absent myometrial invasion), resulting in a favourable 5-year, disease-specific survival rate. ${ }^{25,37}$ This profile is often correlated to oestrogen-progesterone receptor positive tumours and, as consequence, progestin therapies, mostly medroxyprogesterone acetate and megestrol acetate, ${ }^{37}$ are used as conservative treatments. The best strategy, before hormonal therapy, is to proceed to dilatation and curettage, associated with a laparoscopic evaluation of adnexa and peritoneal cavity. ${ }^{38}$ Myometrial invasion must be determined by magnetic resonance imaging before dilatation and curretage.

For some investigators, this hormonal therapy can be combined with local surgical excision by hysteroscopy. The data about this novel surgical approach are still limited, and just a few studies have been published. In 2009, Mazzon et al. ${ }^{39}$ published a study of six women with stage IA endometrial cancer undergoing conservative treatment with a three-step resectoscopic technique. ${ }^{39}$ Step 1 , removal of the tumour; step 2, removal the endometrium adjacent to the tumour; and step 3, removal the underlying myometrium. The operative hysteroscopy was carried out with the women under general anaesthesia. The cervix was dilated to $10 \mathrm{~mm}$ with Hegar's dilator, and a 9-mm resectoscope with $0 \%$ lens was introduced. The uterus was distended with glycine solution under gravity inflow of $70 \mathrm{~mm} \mathrm{Hg}$ pressure. Irrigant fluid was collected and monitored carefully. A 5-mm cutting loop electrode and $100 \mathrm{~W}$ of pure cutting output power were used to resect the tumour lesion, the endometrium near the lesion, and the myometrium under lesion. This three-step hysteroscopic technique was characterised by three different pathologic analysis. If results of the first pathologic analysis was positive (neoplastic lesion) and the other two was negative, megestrol acetate was given daily for 6 months. Instead, if the results of the biopsy of the endometrium near the lesion or the myometrium under the lesion was positive, the women were referred for a total hysterectomy. Women were followed with transvaginal ultrasound and diagnostic hysteroscopy every 3 months during the first year. Four out of six women had subsequent successful pregnancies.

Recently, using a very similar procedure, Laurelli et al. ${ }^{39}$ described 14 women with The International Federation of Gynecology and Obstetrics stage IA (intramucous) endometrial cancer wishing to preserve their fertility. ${ }^{39}$ All 14 women were treated by hystero-resection combined with hormonal therapy (oral or intra-uterine device), after rule out extra-uterine disease. After a minimum 13-month follow up (median 40 months; range 13-79 months), local recurrence was been detected in one women 5 months from hysteroscopic treatment. Three women have attempted to conceive. One of them conceived and delivered a healthy baby at term 14 months from the end of follow up.

This procedure, however, should be carried out with caution because of possible complications of hysteroscopy, and also because of hysteroscopic dissemination of endometrial carcinoma through the fallopian tube during the procedure.

\section{Ovarian cancer}

According to The Federation of Gynaecology and Obstetrics guidelines, current treatment for early epithelial ovarian cancer consists of a maximal cytoreductive surgery, including total abdominal hysterectomy, bilateral salpingo-ovariectomy, pelvic and para-aortic lymphadenectomy, omentectomy, multiple peritoneal biopsy and peritoneal washing. Consequently, fertility is completely destroyed. Because women with early stage disease have an excellent prognosis, the question of quality of life and fertility can become a great concern to them.

Fertility-sparing procedures are also recommended for young women presenting with nonepithelial ovarian cancer (e.g. germ-cell tumours) and epithelial borderline tumour confined to a single ovary. Some investigators have studied this strategy in women with invasive epithelial (EOC) ovarian cancer. In 2005, Morice et al. ${ }^{40}$ described 34 women who underwent fertility procedure for EOC. Thirteen women had stage IA grade 1 disease, 14 had stage IA grade 2, three had stage IA grade 3, three had stage IC and one woman presented with IIA disease. Disease in 11 women recurred, and 10 pregnancies were reported in nine women. Only one woman had disease recurrence among women 
with stage IA grade. The investigators concluded that this strategy is safe and efficient for women with IA grade 1 disease who wish to preserve their fertility. Data on conservative management of EOC are still limited; however, the oncologic safety of fertility-sparing procedures in early EOC has been confirmed. For some investigators, fertility-sparing procedures were found to be safe in women with more advanced stage disease, until stage IC. ${ }^{41}$

In 2008, Park et al. ${ }^{41}$ studied 62 women treated with fertility-sparing procedures. Five-year diseasefree survival and overall survival of women with stage IA disease were $83 \%$ and $91 \%$, respectively, and $78 \%$ and $88 \%$ for women with IC disease, rates comparable to the survival rates of women with stages IA and IC ovarian cancer treated with more radical surgery. ${ }^{41}$ At the end of this study, 19 women had attempted to conceive and 15 had succeeded. Twelve of the 15 women who had become pregnant had received chemotherapy. Twenty-two pregnancies were observed with no congenital anomaly. The investigators concluded that fertility-sparing surgery is a safe treatment option in women with stages IA-IC and grade 1-2 invasive ovarian cancer who wish to preserve reproductive function. According to the number of pregnancies, the reproductive outcomes of this strategy were promising, even in cases of chemotherapy.

Technically, fertility-sparing procedures in ovarian cancer consist of complete surgical staging, including unilateral salpingo-ovariectomy, omentectomy, pelvic and para-aortic lymph-node dissection, peritoneal washing, and peritoneal biopsies. Dilatation and curettage is an obligatory step to identify a possible endometrial localisation. Biopsy of controlateral ovary is not useful, but a careful examination must be carried out. Cystectomy must be carried out in the case of benign-appearing ovarian cysts. In the study by Park et al., ${ }^{41}$ nine women underwent cystectomies to remove benignappearing cysts in the contralateral ovary. They found two bilateral cancers.

Spillage, rupture or morcellation must be avoided when managing ovarian masses or cysts. Midline laparotomy is the standard, but a laparoscopic approach has also been adapted for this procedure.

Fertility-preserving procedures in cases of borderline ovarian tumour is now well-established. Because this type of lesion is often diagnosed in young women, fertility issues are primordial. Given the less aggressive behaviour of borderline tumours, conservative surgery is appropriate in women who wish to preserve their fertility. The standard of care in young women is in fact unilateral salpingoovariectomy, omentectomy and peritoneal washing. Careful examination of the peritoneal surface and contralateral ovary must be conducted, and appendicectomy is indicated in cases of mucinous borderline tumours. Key to diagnosis is pathological analysis in order to avoid any infiltrativemicropapillary pattern. Prognosis of borderline tumour is excellent, with overall survival rates for early stage as high as 97\%. Rates of recurrence are higher in cases of conservative surgery than with radical surgery. Morice et al., ${ }^{42}$ in a retrospective study of 174 women who underwent conservative or radical surgery for borderline tumour, reported rates of recurrence of $5.7 \%, 15.1 \%$, and $36,3 \%$ after radical surgery, adnexectomy and cystectomy, respectively. Recurrence of borderline tumour is rarely in the form of invasive carcinoma. Even if conservative surgery increases the risk for recurrence, disease is often amenable to surgical management, and patient survival is not affected by the conservative approach.

Recent studies have described a novel prophylactic strategy for women with BRCA mutation. In order to preserve hormonal or fertility function in young women undergoing risk-reducing surgery, Leblanc et al. ${ }^{43}$ proposed temporary surgery based on recent knowledge of ovarian carcinogenesis. Given an earlier onset of ovarian cancer observed in cases of BRCA1 mutation, prophylactic surgery is recommended at a younger age in BRCA1 than in BRCA2 mutation carriers. An approach proposed by Leblanc et al. $^{43}$ is a compromise between radical risk reduction surgery and hormonal and fertility preservation before the menopause. The investigators proposed the concept of a radical fimbriectomy. This new technique consists of resecting the fallopian tube from uterine level to the ovary, resecting the totality of the terminal part of the tube or fimbria along with its attachment to the underlying ovary. ${ }^{43}$ The main object of this approach is to remove the source of possible dysplastic cells from the tube and fimbrio-ovarian junction, which can be a possible cause of high-serous carcinoma, while preserving fertility. The first results described by Leblanc et al. ${ }^{43}$ in a preliminary study are promising. In their prospective study of 14 women, the investigators carried out a radical fimbriectomy, then a resection of the remaining ovaries. Radical fimbriectomy and residual ovary were sent for pathological evaluation in order to assess cellular abnormalities from each part of the specimen (carcinomas, serous tubal intraepithelial carcinoma, p53 signature and ki67). Despite lesions found on the fimbria or on the 
attached part of the ovary, no abnormality was found in the remaining part of ovaries. The investigators concluded that this procedure could be a temporary option for women with BRCA mutation who wish to preserve fertility. Further studies are required.

\section{Conclusion}

Fertility-sparing treatments have been successfully attempted in selected cases of cervical, endometrial and ovarian cancer, and gynaecologists should be familiar with fertilitypreserving options in women with gynaecological malignancies. Options to preserve fertility include shielding to reduce radiation damage, fertility preservation when undergoing cytotoxic treatments, cryopreservation, assisted reproduction techniques, and fertility-sparing surgical procedures. Radical vaginal trachelectomy with laparoscopic lymphadenectomy is an oncologically safe, fertility-preserving procedure. It has been accepted worldwide as a surgical treatment of small early stage cervical cancers. Selected cases of early stage ovarian cancer can be treated by unilateral salpingo-ophorectomy and surgical staging. Hysteroscopic resection and progesterone treatment are used in young women who have endometrial cancer to maintain fertility and avoid surgical menopause. Appropriate patient selection, and careful oncologic, psychologic, reproductive and obstetric counselling, is mandatory.

\section{Conflicts of interest}

None declared.

\section{Practice points}

- Radical trachelectomy is a feasible, safe, and accepted surgical procedure in women with small cervical cancer lesions, and should be considered in young women desiring fertility preservation.

- Neoadjuvant chemotherapy followed by conservative fertility-sparing surgery is a nonstandardised option for women with bulky cervical tumours.

- A conservative surgical approach after neoadjuvant chemotherapy for cervical cancer should be abandoned in cases of poor chemotherapy response.

- In case of conservative strategy for early endometrial cancer, laparoscopic examination of abdominal cavity is a primordial step of pre-therapeutic evaluation.

- Combined operative hysteroscopy and progestin therapy have a role in selected women desiring to preserve fertility.

- Unilateral adnexectomy can be considered as a treatment option in women with stage I epithelial ovarian cancer who desire childbearing.

- Patient commitment to close follow up is essential after a surgical-sparing procedure.

- Ovarian transposition should be considered in young women undergoing pelvic radiation therapy.

- Fertility outcome after conservative management is promising and, in today's era of reproductive technology, this option must be presented to woman as part of her oncologic plan of care.

\section{Research agenda}

- Safety of simple trachelectomy and cone biopsy with or without neoadjuvant chemotherapy.

- Effectiveness of other less gonadotoxic regimens for neoadjuvant chemotherapy in women with early stage cervical cancer desiring to preserve fertility.

- Oncological safety of radical fimbriectomy in BRCA mutation carriers. 


\section{References}

*1. Plante M. Vaginal radical trachelectomy: an update. Gynecol Oncol 2008; 111: S105-S110.

2. Speiser D, Mangler M, Kohler C et al. Fertility outcome after radical vaginal trachelectomy: a prospective study of 212 patients. Int J Gynecol Cancer 2011; 21: 1635-1639.

3. Hertel H, Kohler C, Grund D et al. Radical vaginal trachelectomy (RVT) combined with laparoscopic pelvic lymphadenectomy: prospective multicenter study of 100 patients with early cervical cancer. Gynecol Oncol 2006; 103: 506-511.

4. Ungar L, Palfalvi L, Hogg R et al. Abdominal radical trachelectomy: a fertility-preserving option for women with early cervical cancer. BJOG 2005; 112: 366-369.

5. Gien LT \& Covens A. Fertility-sparing options for early stage cervical cancer. Gynecol Oncol 2010; 117: 350-357.

6. Beiner ME \& Covens A. Surgery insight: radical vaginal trachelectomy as a method of fertility preservation for cervical cancer. Nat Clin Pract Oncol 2007; 4: 353-361.

7. Dursun P, LeBlanc E \& Nogueira MC. Radical vaginal trachelectomy (Dargent's operation): a critical review of the literature. Eur J Surg Oncol 2007; 33: 933-941.

*8. Dargent D, Martin X, Sacchetoni A et al. Laparoscopic vaginal radical trachelectomy: a treatment to preserve the fertility of cervical carcinoma patients. Cancer 2000; 88: 1877-1882.

*9. Abu-Rustum NR, Sonoda Y, Black D et al. Fertility-sparing radical abdominal trachelectomy for cervical carcinoma: technique and review of the literature. Gynecol Oncol 2006; 103: 807-813.

10. Kim JH, Park JY, Kim DY et al. Fertility-sparing laparoscopic radical trachelectomy for young women with early stage cervical cancer. BJOG 2010; 117: 340-347.

11. Martin A \& Torrent A. Laparoscopic nerve-sparing radical trachelectomy: surgical technique and outcome. J Minim Invasive Gynecol 2010; 17: 37-41.

12. Geisler JP, Orr CJ \& Manahan KJ. Robotically assisted total laparoscopic radical trachelectomy for fertility sparing in stage IB1 adenosarcoma of the cervix. J Laparoendosc Adv Surg Tech A 2008; 18: 727-729.

13. Persson J, Kannisto P \& Bossmar T. Robot-assisted abdominal laparoscopic radical trachelectomy. Gynecol Oncol 2008; 111: 564-567.

14. Ramirez PT, Schmeler KM, Malpica A et al. Safety and feasibility of robotic radical trachelectomy in patients with earlystage cervical cancer. Gynecol Oncol 2010; 116: 512-515.

15. Sonoda Y, Papageorgiou T, Taieb S et al. Radical trachelectomy via a trans-sacral approach: a case report. Gynecol Oncol 2003; 90: 466-470.

16. Marchiole P, Tigaud JD, Costantini S et al. Neoadjuvant chemotherapy and vaginal radical trachelectomy for fertilitysparing treatment in women affected by cervical cancer (FIGO stage IB-IIA1). Gynecol Oncol 2011; 122: 484-490.

*17. Maneo A, Chiari S, Bonazzi C et al. Neoadjuvant chemotherapy and conservative surgery for stage IB1 cervical cancer. Gynecol Oncol 2008; 111: 438-443.

18. Covens A, Rosen B, Murphy J et al. How important is removal of the parametrium at surgery for carcinoma of the cervix? Gynecol Oncol 2002; 84: 145-149.

19. Landoni F, Parma G, Peiretti M et al. Chemo-conization in early cervical cancer. Gynecol Oncol 2007; 107: S125-S126.

20. Plante M, Lau S, Brydon L et al. Neoadjuvant chemotherapy followed by vaginal radical trachelectomy in bulky stage IB1 cervical cancer: case report. Gynecol Oncol 2006; 101: 367-370.

*21. Morice P, Castaigne D, Haie-Meder C et al. Laparoscopic ovarian transposition for pelvic malignancies: indications and functional outcomes. Fertil Steril 1998; 70: 956-960.

22. Prouvost MA, Canis M, Le Bouedec G et al. Ovarian transposition with per-celioscopy before curietherapy in stage IA and IB cancer of the uterine cervix [in French]. J Gynecol Obstet Biol Reprod (Paris) 1991; 20: 361-365.

23. Williams RS \& Mendenhall N. Laparoscopic oophoropexy for preservation of ovarian function before pelvic node irradiation. Obstet Gynecol 1992; 80: 541-543.

24. Ray GR, Trueblood HW, Enright LP et al. Oophoropexy: a means of preserving ovarian function following pelvic megavoltage radiotherapy for Hodgkin's disease. Radiology 1970; 96: 175-180.

25. Belinson JL, Doherty M \& McDay JB. A new technique for ovarian transposition. Surg Gynecol Obstet 1984; 159: 157-160.

26. Gaetini A, De Simone M, Urgesi A et al. Lateral high abdominal ovariopexy: an original surgical technique for protection of the ovaries during curative radiotherapy for Hodgkin's disease. J Surg Oncol 1988; 39: 22-28.

27. Hodel K, Rich WM, Austin P et al. The role of ovarian transposition in conservation of ovarian function in radical hysterectomy followed by pelvic radiation. Gynecol Oncol 1982; 13: 195-202.

28. Rasool N \& Rose PG. Fertility-preserving surgical procedures for patients with gynecologic malignancies. Clin Obstet Gynecol 2010; 53: 804-814.

29. Eitan R, Krissi H, Beller U et al. Laparoscopic adnexal transposition: novel surgical technique. Int J Gynecol Cancer 2011; 21: 1704-1707.

30. Morice P, Juncker L, Rey A et al. Ovarian transposition for patients with cervical carcinoma treated by radiosurgical combination. Fertil Steril 2000; 74: 743-748.

*31. Querleu D, Gladieff L, Delannes M et al. Preservation of fertility in gynaecologic cancers [in French]. Bull Cancer 2008; 95: 487-494.

32. Kim YB, Holschneider $\mathrm{CH}$, Ghosh K et al. Progestin alone as primary treatment of endometrial carcinoma in premenopausal women. Report of seven cases and review of the literature. Cancer 1997; 79: 320-327.

33. Niwa K, Tagami K, Lian $\mathrm{Z}$ et al. Outcome of fertility-preserving treatment in young women with endometrial carcinomas. BJOG 2005; 112: 317-320.

34. Vinker S, Shani A, Open M et al. Conservative treatment of adenocarcinoma of the endometrium in young patients. Is it appropriate? Eur J Obstet Gynecol Reprod Biol 1999; 83: 63-65.

35. Lee TS, Kim JW, Kim TJ et al. Ovarian preservation during the surgical treatment of early stage endometrial cancer: a nation-wide study conducted by the Korean Gynecologic Oncology Group. Gynecol Oncol 2009; 115: 26-31.

36. Poilblanc M, Samouelian V \& Querleu D. Ovarian preservation during treatment of early stage endometrial cancer. Bull Cancer 2012; 99: 61-68. 
*37. Ramirez PT, Frumovitz M, Bodurka DC et al. Hormonal therapy for the management of grade 1 endometrial adenocarcinoma: a literature review. Gynecol Oncol 2004; 95: 133-138.

38. Morice P, Fourchotte V, Sideris L et al. A need for laparoscopic evaluation of patients with endometrial carcinoma selected for conservative treatment. Gynecol Oncol 2005; 96: 245-248.

39. Mazzon I, Corrado G, Masciullo V et al. Conservative surgical management of stage IA endometrial carcinoma for fertility preservation. Fertil Steril 2010; 93: 1286-1289.

*40. Morice P, LeBlanc E, Rey A et al. Conservative treatment in epithelial ovarian cancer: results of a multicentre study of the GCCLCC (Groupe des Chirurgiens de Centre de Lutte Contre le Cancer) and SFOG (Societe Francaise d'Oncologie Gynecologique). Hum Reprod 2005; 20: 1379-1385.

41. Park JY, Kim DY, Suh DS et al. Outcomes of fertility-sparing surgery for invasive epithelial ovarian cancer: oncologic safety and reproductive outcomes. Gynecol Oncol 2008; 110: 345-353.

42. Morice P, Camatte S, El Hassan J et al. Clinical outcomes and fertility after conservative treatment of ovarian borderline tumors. Fertil Steril 2001; 75: 92-96.

*43. LeBlanc E, Narducci F, Farre I et al. Radical fimbriectomy: a reasonable temporary risk-reducing surgery for selected women with a germ line mutation of BRCA 1 or 2 genes? Rationale and preliminary development. Gynecol Oncol 2011; 121: $472-476$.

*44. Dargent D, Brun JL \& Roy M. La trachelectomie élargie (T.E). Une alternative à l'hysterectomie radicale dans le traitement des cancers infiltrants developpes sur la face externe du col uterin. J Obstet Gynecol 1994; 2: 292-295. 